\title{
Editorial
}

\section{Human Embryonic Stem Cells: A Resource for In Vitro Neuroscience Research?}

\author{
\begin{tabular}{l}
\hline Trevor R Norman' \\
'Department of Psychiatry, University of Melbourne, Austin Hospital, Heidelberg, VIC, Australia \\
\hline
\end{tabular} \\ Neuropsychopharmacology (2006) 31, 257I-2572. doi:I0. I038/sj.npp. I 30 I 126
}

\begin{abstract}
'Modern neuroscience has, in effect, lifted the hood and permitted us to peer, however tentatively, at the engine'. Francis Fukuyama, Our Post-human Future
\end{abstract}

Notwithstanding the ethical debate surrounding the use of human embryonic stem cells (hESCs), there can be no doubt that such cells hold promise for the development of treatments for a range of neurodegenerative disorders, even if the reality is more sober (Scott, 2006). These pluripotent cells can, under the right conditions, be programmed to differentiate into neural and other tissue-specific cells (Gokhan and Mehler, 2001). Perhaps more importantly, hESCs can be used to study the processes involved in the early stages of neurodevelopment. It is already apparent that some neurodegenerative disorders may be disorders of neural development (Gokhan and Mehler, 2001) or adult cellular plasticity (van Dellen et al, 2005). Protein characteristics of disease processes, such as $\alpha$-synuclein (Parkinson disease), huntingtin (Huntington's disease), and pre-senilin-1 (Alzheimer's disease), may interact with each other and normal protein partners to cause sublethal changes in cellular homeostasis. The timing of their onset during the process of neurodevelopment, or the way they disrupt adult cellular plasticity, may well be critical in determining patterns of illness expression.

In this issue of the journal, Zeng and co-workers report another use of hESCs: to study in vitro neurotoxicity of chemicals. Why hESCs and not adult neural stem cells or indeed embryonic stem cells derived from other species? The answer probably lies with the nature of the hESCs themselves - they are pluripotent. It is not clear that all adult stem cells are capable of differentiation into neurones, whereas neural stem cells have limited capacity to generate functional neurones (Bjorklund and Lindvall, 2000). Stem cells from other species may be inappropriate owing to demonstrable differences in the in vivo toxicity of the compound examined, the neurotoxin 1-methyl-4-phenylpyridine $\left(\mathrm{MPP}^{+}\right)$oxidatively derived from 1-methyl-4phenyl-1,2,3,6-tetrahydropyridine (MPTP). The use of hESCs suggests that the data obtained are directly applicable to the in vivo situation, although like all in vitro models, there are inherent limitations.
The study examined in vitro the toxicity of $\mathrm{MPP}^{+}$in hESCs, which were differentiated into dopaminergic neurons. After 3 weeks, the hESC colonies stained positive for tyrosine hydroxylase and on microscopic examination process formation was observed. This provided evidence that the cells were indeed a model of dopamine neurones, although the ultimate functional test of this would be to demonstrate dopamine release. $\mathrm{MPP}^{+}$is well known to cause Parkinsonian symptoms in some animal species. Not surprisingly, administration of the compound caused a dose-dependent diminution in the number of dopaminergic neurones in culture (measured by staining for tyrosine hydroxylase and release of lactate dehydrogenase into the medium). Investigating the mechanism of the effect, the authors concluded that cell loss was probably preceded by the generation of reactive oxygen species. The data are more than likely to be relevant to the in vivo situation, as a similar dopaminergic neuronal loss occurs in animals after administration of the precursor MPTP. More importantly, cell death in the culture could be prevented by preincubation of the neurones with glial-derived neurotrophic factor (GDNF). Whether GDNF would also afford protection against the neurotoxic effects of $\mathrm{MPP}^{+}$in vivo remains to be tested. These findings are perhaps not surprising. Although many questions are raised by the results presented, the study represents a methodology that can be utilized for the examination of the potential neurotoxic effects of many compounds in isolated dopaminergic neuronal systems. Clearly, some questions may not be answerable within the framework of this methodology. For example, can the addition of GDNF (or other neuroprotective principles) reverse the damage done to an already diseased neurone? Nevertheless, the experiments described provide a robust means to examine the role of toxins suspected in the environmental etiology of Parkinson's disease, for example, paraquat. Although not examined in this report, the method may well be applicable to other appropriately differentiated neuronal systems such as the serotonergic system. Recent concern about neuronal damage resulting from the prolonged use of so-called 'party drugs' such as ecstasy could be examined in such a model. A detailed assessment of the neurochemical processes 
involved would also be accessible. However, if the appropriate neurotransmitter, receptors, transporters, and synaptic connections are not expressed in this in vitro model, its applications may be limited.

The ethical justification for the use of hESCs has been that there is the potential to examine possible preventative treatments for various diseases in vitro. Further, the details of the neurochemical processes involved in such toxicity might potentially be teased apart. The present paper represents a practical example of the use of hESCs in this light. It would also seem that the hESCs could be engineered in such a way as to express abnormal proteins associated with neurodegenerative illnesses and to examine the effects of putative therapeutic agents in this context. Clearly, in genetically determined disorders, such as Huntington's disease, non-human stem cells from mouse models could be used in this manner to provide further insights into this debilitating illness. However, evidence of disrupted adult neurogenesis that can be pharmacologically ameliorated in
Huntington's transgenic mice (Grote et al, 2005) suggests that modulation of adult stem cells may also be a promising therapeutic target for such neurodegenerative diseases.

\section{REFERENCES}

Bjorklund A, Lindvall O (2000). Cell replacement therapies for central nervous system disorders. Nat Neurosci 3: 537-544.

Gokhan S, Mehler MF (2001). Basic and clinical neuroscience applications of embryonic stem cells. Anatom Rec 265: 142-156.

Grote HE, Bull ND, Howard ML, van Dellen A, Blakemore C, Bartlett PF et al (2005). Cognitive disorders and neurogenesis deficits in Huntington's disease mice are rescued by fluoxetine. Eur J Neurosci 22: 2081-2088.

Scott CT (2006). Stem Cell Now. From the Experiment that Shook the World to the New Politics of Life. Pi Press: New York.

van Dellen A, Grote HE, Hannan AJ (2005). Gene-environment interactions, neuronal dysfunction and pathological plasticity in Huntington's disease. Clin Exp Pharmacol Physiol 32: 1007-1019. 